\section{Cardiovascular magnetic resonance in rare systemic diseases}

\author{
Antonello D'Andrea, ${ }^{1}$ Marianna Fontana, ${ }^{2}$ \\ Rosangela Cocchia, ${ }^{1}$ Raffaele Calabrò, ${ }^{1}$ \\ Maria Giovanna Russo, ${ }^{1}$ \\ James C. Moon 2,3 \\ 'Monaldi Hospital, Second University \\ of Naples, AORN Ospedali dei Colli, Naples, \\ Italy; ${ }^{2}$ Heart Hospital Imaging Centre, Heart \\ Hospital, London; ${ }^{3}$ Institute of \\ Cardiovascular Sciences, UCL, London, UK
}

\section{Abstract}

The heart may be involved in a number of systemic syndromes. The pericardium, myocardium, heart valves, and coronary arteries may be involved either singly or in various combinations. In most cases the cardiac manifestations are not the dominant feature, but in some it is the primary determinant of symptoms and survival. Both the early identification of cardiac involvement and the etiology underneath is of paramount importance, as some causes require specific treatment and may be correctable. In this respect non-invasive imaging plays a central role especially in the context of rare cardiac disease, where specific imaging features can help to make the appropriate diagnosis on a substantial proportion of them, enabling the physician to choose the best management strategy tailored to the disease. Whereas echocardiography is the firstline investigation for detecting a cardiac involvement in systemic disease, cardiovascular magnetic resonance (CMR) provides additional incremental data allowing in addition to a detailed examination of cardiac structure and function also the tissue characterization. The aim of this review is therefore to delineate the role of CMR in detecting cardiac involvement in patients with rare systemic diseases and delineate the specific imaging features of the different etiologies.

\section{Introduction}

The heart may be involved in a number of systemic syndromes. The pericardium, myocardium, heart valves, and coronary arteries may be involved either singly or in various combinations. In most cases the cardiac manifestations are not the dominant feature, but in some it is the primary determinant of symptoms and survival.

Both the early identification of cardiac involvement and the etiology underneath is of paramount importance, as some causes require specific treatment and may be correctable. In this respect non-invasive imaging plays a central role especially in the context of rare cardiac disease, where specific imaging features can help to make the appropriate diagnosis on a substantial proportion of them, enabling the physician to choose the best management strategy tailored to the disease.

Imaging cardiac involvement in systemic disease is challenging and requires a multimodality approach. Echocardiography is the first-line investigation; however, it is limited by poor image quality in some patients and gives very little information about tissue characteristics. Cardiovascular magnetic resonance (CMR) provides additional incremental data in patients with systemic disease. The excellent image quality obtained by CMR allows a detailed examination of cardiac structure and function. However the key advantage, unique to this imaging modality, that has revolutionized the role of CMR in the evaluation of cardiac disease, is the tissue characterization. CMR is excellent for distinguishing different soft tissues based on their magnetic properties described by the relaxations times $\mathrm{T} 1$, T2, and $\mathrm{T} 2 *$. Normal and abnormal myocardium may have different relaxation times, but the addition of a gadolinium contrast agent greatly magnifies these differences. Gadolinium (Gd) is chelated and behaves as a passive, extravascular, extracellular contrast agent. After an intravenous bolus, three time phases are considered. The first pass can be used for perfusion imaging and is often given during vasodilator stress to detect ischemia. Early after contrast, severely hypovascular regions will not enhance (thrombus or microvascular obstruction in acute myocardial infarction). In the late phase (5 min plus post bolus), contrast lingers in areas of infarction or focal fibrosis due to slower contrast kinetics and a greater volume of distribution in extracellular water associated with collagen. Early after the first pass of Gd, a significant fraction of the injected Gd enters the interstitial space. Indeed, several minutes after intravenous administration of $\mathrm{Gd}$, the larger volume of distribution available in necrotic or fibrotic myocardium results in a higher concentration of contrast agent than what is present in viable myocardium. This is typically referred to as late gadolinium enhancement (LGE). ${ }^{1}$ This can be assessed either visually or quantitatively based on relative enhancement compared to the background. ${ }^{2}$ The extent and pattern of LGE varies according to the underlying disease process and is frequently of prognostic significance and can be diagnostic of the underlying etiology.

In order to improve sensitivity in detection of interstitial diffuse fibrosis, T1 mapping
Correspondence: Antonello D'Andrea, via Michelangelo Schipa 44, 80122 Naples, Italy. Tel. +39.081.706.4150 - Fax: +39.081 .714 .5205 . E-mail: antonellodandrea@libero.it

Key words: cardiovascular magnetic resonance, rare systemic diseases, tissue characterization.

Received for publication: 20 May 2013.

Revision received: 10 October 2013.

Accepted for publication: 11 October 2013.

This work is licensed under a Creative Commons Attribution NonCommercial 3.0 License (CC BYNC 3.0).

(C) Copyright A. D'Andrea et al., 2013

Licensee PAGEPress, Italy

Cardiogenetics 2013; 3:e8

doi:10.4081/cardiogenetics.2013.e8

technique have been recently proposed to quantifying $\mathrm{T} 1$ values for each voxel in the myocardium. This approach allows generating a parametric map without the need to compare it to a normal reference standard before or after the use of a contrast agent. $^{3}$ At the moment the applicability of this method in different setting in still to standardize.

Currently, a comprehensive CMR protocol including anatomy, function, and LGE imaging is able to identify the cardiac involvement and the underlying etiology in a substantial proportion of patients with systemic disease.

The aim of this review is therefore to delineate the role of CMR in detecting cardiac involvement in patients with rare systemic diseases and delineate the specific imaging features of the different etiologies.

We identified a list of rare diseases, defined as disease with a less than 1 in 2000 people prevalence, in which cardiac involvement is frequently documented.

\section{Sarcoidosis}

Myocardial involvement occurs in $20-60 \%$ of patients with sarcoidosis at autopsy. ${ }^{4}$ However, only $5 \%$ of patients have signs or symptoms of cardiac involvement, ${ }^{5,6}$ making diagnosis of cardiac sarcoidosis an exceptional challenge. Cardiac arrhythmias and progressive congestive $\mathrm{HF}$ are the most frequent cause of death in patients with cardiac sarcoidosis. ${ }^{7,8}$ Thus, it is important to identify the presence of cardiac involvement in these patients who may benefit from medical and/or implantable defibrillator therapy.

CMR can show some of the features of cardiac sarcoid as septal thinning, left ventricle/ right ventricle (LV/RV) dilation, systolic dysfunction or pericardial effusion. ${ }^{7,9}$ However 
these structural and functional changes are non specific, as they can be found in different type of cardiac diseases. Several studies have shown the usefulness of LGE-CMR in the detection of characteristic features of cardiac sarcoidosis and evaluation of response to steroid treatment. ${ }^{10,11}$ The LGE in these patients usually shows a non-ischemic pattern with hyper-enhancement of the mid-myocardial wall or the epicardium in an unpredictable distribution $^{12}$ (Figure 1 and Table 1). The anteroseptal and inferolateral walls are most frequently involved, although sometimes hyper-enhancement is seen in other territories, including the RV. ${ }^{13}$ Follow-up studies showed that the enhanced areas were notably diminished in size and intensity after steroid treatment. ${ }^{14}$ The LGE-CMR scan can also be used to guide endomyocardial biopsy in sarcoidosis. Additionally,T2-weighted sequences may monitor disease activity by the identification of edematous areas associated with inflammation and granulomatous lesions. ${ }^{15}$ Recently, myocardial delayed enhancement was correlated to a higher rate of adverse events including cardiac death. ${ }^{16}$

\section{Amyloidosis}

Cardiac involvement in amyloidosis is characterized by fibril deposition within the myocardium causing a concentric wall thickening with severe systo-diastolic dysfunction. Cardiac involvement is common in patients with both amyloid light-chain (AL) and transttiretin amyloidosis. The heart in AL amyloidosis (the most common form and with the worse prognosis) is affected in up to $50 \%$ of cases, and congestive heart failure (HF) is the presenting clinical manifestation in about onehalf of these patients. ${ }^{17}$ Once congestive HF occurs, the median survival is $<6$ months in untreated patients; therefore, early recognition of the disease and prompt initiation of therapy is critical.

The tissue characterization with cardiac CMR plays a central role in the diagnosis of cardiac amyloidosis. After the administration of the contrast, the presence of amyloid protein in the heart results in accelerated removal of gadolinium from the blood and increased myocardial uptake, such that more gadolinium is present in the myocardium than the blood pool. ${ }^{18}$ This change in gadolinium kinetic behaviour is almost unique to amyloidosis. There is a unique pattern of late circumferential enhancement of the subendocardium of the $\mathrm{LV}$ and RV.

With subendocardial enhancement of the LV and RV endocardium, there may be a characteristic zebra-stripe appearance sparing the midwall of the interventricular septum. ${ }^{18-20}$ The blood pool appears typically dark, reflecting high myocardial contrast uptake and fast blood pool washout (Figure 1). These characteristic changes may help to detect cardiac amyloidosis also in patients with normal cardiac structure and function by echocardiography. ${ }^{21}$ Recently non contrast techniques using T1 mapping have been shown to have a high diagnostic accuracy for detecting cardiac AL amyloidosis with potential to be more sensitive for detecting early disease than LGE imaging ${ }^{22}$ (Figure 2). Additionally a recent study proved the potential of CMR to become the first non invasive technique able to measure the cardiac amyloid burden, raising the possibility that CMR may provide a much needed cardiac surrogate endpoint for the various promising new therapies for amyloidosis currently in preclini-

Table 1. Myocardial tissue characterization using cardiovascular magnetic resonance.

\begin{tabular}{|c|c|c|c|}
\hline Technique & Tissue characterization & Disease & Typical features \\
\hline \multicolumn{4}{|c|}{ Non-contrast } \\
\hline T1 weighted spin echo & Increased fat content & $\begin{array}{l}\text { ARVC/D } \\
\text { Cardiac masses }\end{array}$ & - \\
\hline T2 weighted spin echo & Increased water content & $\begin{array}{l}\text { Acute infarction } \\
\text { Acute myocarditis }\end{array}$ & - \\
\hline T2* weighted sequences & Iron & Hemocromatosis & - \\
\hline \multicolumn{4}{|c|}{ Contrast-based } \\
\hline T1 weighted spin echo & $\begin{array}{l}\text { Inflammation } \\
\text { (hyperemia and capillary } \\
\text { leak as early enhancement) }\end{array}$ & $\begin{array}{l}\text { Myocarditis } \\
\text { Acute infarction }\end{array}$ & - \\
\hline \multirow[t]{7}{*}{$\begin{array}{l}\text { T1 weighted/inversion recovery } \\
\text { Late enhancement }\end{array}$} & $\begin{array}{l}\text { Areas with delayed wash out of gadolinium } \\
\text { (fibrosis, necrosis, infiltrative diseases) }\end{array}$ & \multirow[t]{7}{*}{$\begin{array}{l}\text { Myocardial infarction } \\
\text { Amyloidosis }\end{array}$} & $\begin{array}{l}\text { - } \\
\text { Patchy transmural pattern with characteristic } \\
\text { zebra-stripe appearance sparing } \\
\text { the midwall of the interventricular septum }\end{array}$ \\
\hline & Sarcoidosis & & $\begin{array}{l}\text { Anteroseptal and inferolateral mid-myocardial } \\
\text { or epicardium involvement in } \\
\text { an unpredictable distribution }\end{array}$ \\
\hline & Systemic sclerosis & & $\begin{array}{l}\text { Midwall LGE distribution with a linear pattern, } \\
\text { sparing the subendocardium } \\
\text { and epicardium in basal and mid-cavity segments } \\
\text { of the LV }\end{array}$ \\
\hline & Anderson-Fabry disease & & $\begin{array}{l}\text { Predilection for basal inferolateral } \\
\text { LV segments sparing subendocardium }\end{array}$ \\
\hline & Eosinophilic disease & & Exclusive involvement of endomyocardium \\
\hline & Glicogen storage III & & $\begin{array}{l}\text { Extensive multifocal LGE in mid myocardium } \\
\text { and other areas }\end{array}$ \\
\hline & Becker muscular dystrophy & & Extensive mid-myocardial LGE \\
\hline
\end{tabular}

ARVC/D, arrhythmogenic right ventricular cardiomyopathy/dysplasia; LGE, late gadolinium enhancement; LV, left ventricle. 
cal development and early phase clinical trials. ${ }^{23}$ Death is predicted by gadolinium kinetics, with post-gadolinium intramyocardial T1 difference between the subepicardium and subendocardium predicting mortality with $85 \%$ accuracy (the smaller the T1 intramyocardial gradient, the worse the prognosis).Finally, cardiac amyloidosis has been also associated with hypointense signal on T2-weighted images, and a lower T2 ratio was independently associated with shortened survival. ${ }^{24}$

\section{Systemic sclerosis}

Systemic sclerosis (SSc) is a connective tissue disease characterized by vascular pathology, especially of the microvasculature, and tissue fibrosclerosis with involvement of the skin, gastrointestinal tract and organs such as the lungs, heart and kidneys.

During the first five-year period from the onset of the disease, $44 \%$ of deaths had a cardiac or renal origin compared to $18 \%$ in the successive five-year period, with an overall $40 \%$ cardiac mortality. ${ }^{25}$

Cardiac involvement is present in $50-80 \%$ of SSc patients and consists of various pathological changes including hypertrophy, inflammation and myocardial fibrosis, occasionally associated with segmental necrosis of the myocytes and contraction band necrosis due to severe ischemia and reperfusion injury, despite the absence of obstructive coronary lesions.

The usefulness of CMR in SSc has been recently underlined, focusing on LGE abnormalities. ${ }^{26}$ Late enhancement was characteristically midwall, with a linear pattern, sparing the subendocardium and epicardium (Table 1). These linear striae infrequently appeared interrupted or demonstrated small spiculations. Enhancement involved predominantly the basal and mid-cavity segments of the LV, whereas the apical segments were relatively less involved. In addition to a linear pattern, patchy nodular enhancements at the basal segment of the interventricular septum involving the RV lower or upper insertion points and nodular enhancement in the basal segment of the LV free wall were observed ${ }^{27}$ (Figure 1).

Subsequently, Hachulla et al. confirmed that CMR can analyze precisely the different patterns of heart involvement in SSc by differentiating morphological, functional, perfusion and delayed contrast enhancement abnormalities. In this study, patients with limited cutaneous SSc had roughly the same CMR abnormalities as those with diffuse cutaneous SSc, and RV dilatation was not specific for pulmonary arterial hypertension. ${ }^{27}$

\section{Anderson-Fabry disease}

Anderson-Fabry disease (AFD) is an Xlinked disorder of lysosomal metabolism caused by the partial or complete deficiency of the enzyme $\alpha$-galactosidase $\mathrm{A}$, which results in the accumulation of excess cellular glycosphingolipid within the blood vessels and heart. ${ }^{28}$ This is a cause of LV hypertrophy associated with progressive myocardial fibrosis and death from heart failure. CMR can be used to assess LV function, determine the pattern and extent of LV hypertrophy, and identify areas of fibrosis. The LGE pattern typically spares the subendocardium and shows a striking predilection for the basal inferolateral LV segments (Figure 1). Recently Non-contrast $\mathrm{T}_{1}$ mapping techniques have been shown to have a great potential as a unique and powerful measurement in the imaging assessment of Anderson-Fabry disease with $\mathrm{T}_{1}$ able to completely discriminate between AFD and other diseases with no overlap $^{29}$ (Figure 2). Histological data suggest that these areas of LGE correspond to regions of replacement fibrosis. ${ }^{30} \mathrm{Up}$ to $6 \%$ of patients who are initially diagnosed with hypertrophic cardiomyopa- thy actually have evidence of Anderson-Fabry disease. $^{31}$ The implications of an incorrect diagnosis are considerable because patients with Anderson-Fabry disease respond to enzyme replacement therapy, including an improvement in cardiac function and regression of hypertrophy. ${ }^{32,33}$ Ideally, the administration of recombinant $\alpha$-galactosidase A should be started before myocardial fibrosis has developed to achieve long-term improvement in myocardial morphology, function, and exercise capacity. ${ }^{34}$ Anderson-Fabry disease should always be considered if unexplained LV hypertrophy is seen, particularly in a young patient.

\section{Glycogen storage disease type III}

In Glycogen storage disease type III, the functional absence of a glycogen debranching enzyme results in intracellular glycogen accumulation. However, cardiac hypertrophy may also result from interstitial expansion. In a study by Moon et al, gadolinium-diethylenetriaminepenta-acetic acid rest perfusion demonstrated multifocal first pass mid-myocardial defects and late imaging demonstrated hyper-

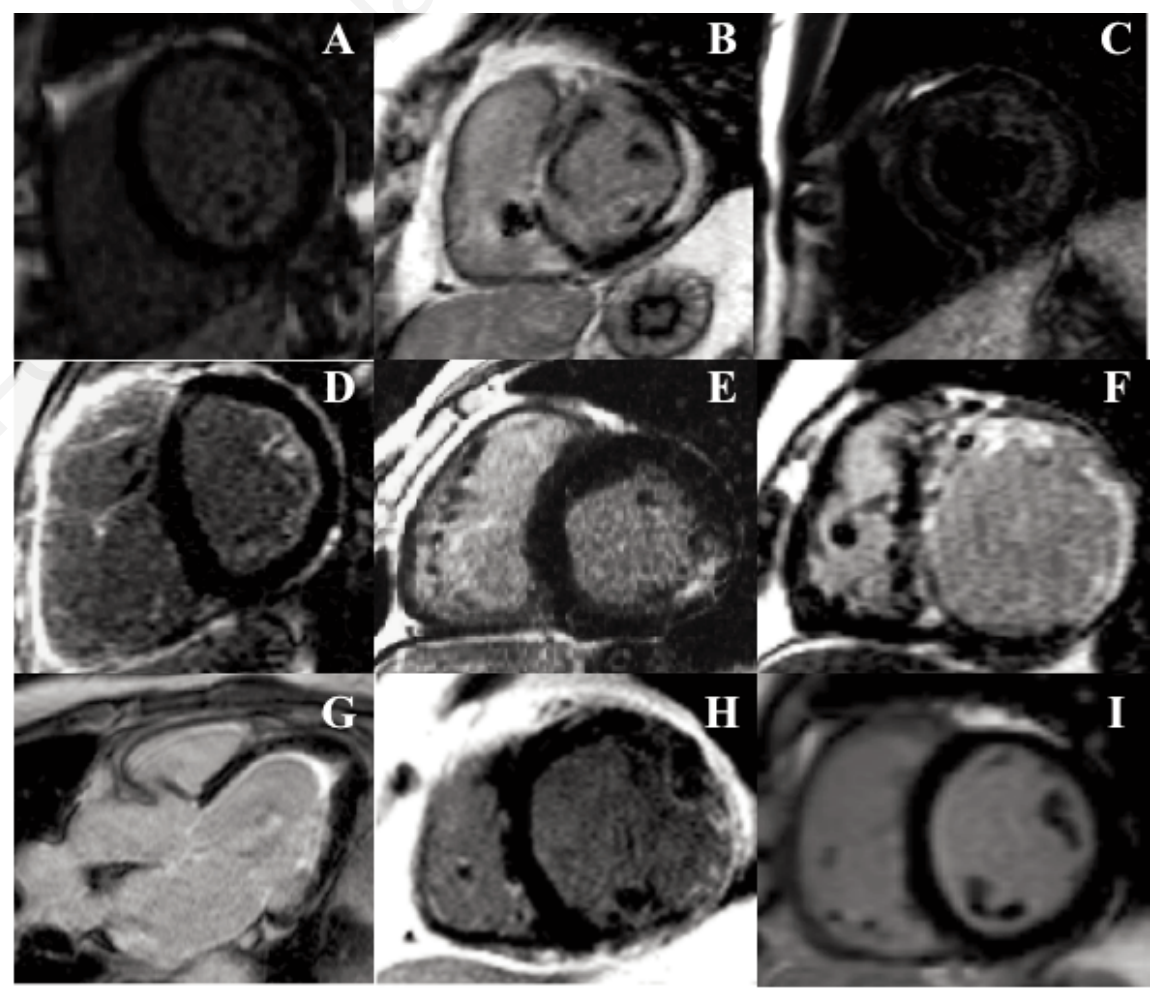

Figure 1. Tissue characterization in different heart diseases. A) Normal individual [no late gadolinium enhancement (LGE)]; B) sarcoid with patchy LGE and pace-maker lead in the right ventricle C) global subendocardial LGE in amyloid; D) systemic sclerosis; E) Anderson-Fabry disease with inferolateral LGE; F) Glycogen storage disease type III with extensive multifocal LGE in mid myocardium and other areas G) apical subendocardial LGE in Churg-Strauss; H) LGE in the inferolateral wall and other areas in Becker muscular dystrophy; I) no LGE in stress-induced (Takotsubo) cardiomyopathy. 


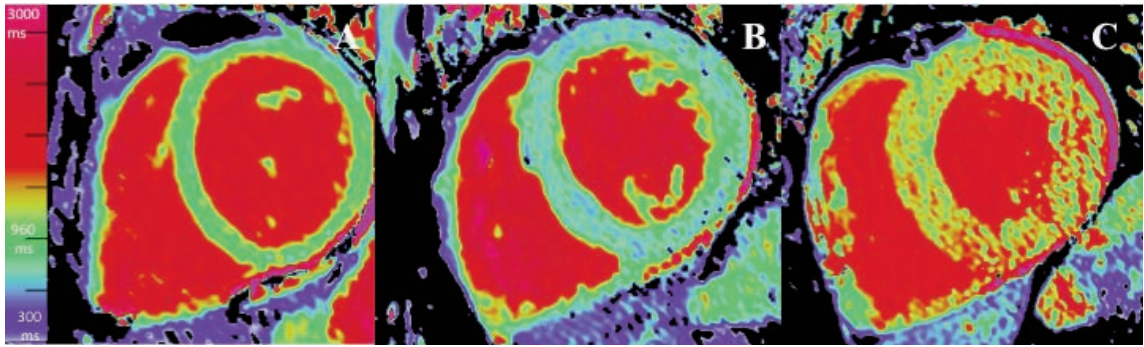

Figure 2. A pre contrast T1 map (ShMOLLI in this case) of the basal short axis from A) a healthy volunteer; B) patient with Anderson-Fabry disease (AFD); and C) a patient with cardiac amyloid. Normal myocardium is green; blue areas (representing lower T1) are seen in the AFD septum, and red (longer T1) are seen in patients with cardiac amyloid.

enhancement in these and other $\operatorname{areas}^{35}$ (Figure 1).

\section{Eosinophilic diseases}

Cardiac hyper-eosinophilia (e.g., malignant, Loefflers; Churgg-Strauss) can cause endomyocardial fibrosis, valve disease and papillary muscle dysfunction, diastolic dysfunction, intracardiac thrombus formation, and heart failure. These features are highly characteristic and well diagnosed by CMR: T2-weighted sequences may monitor disease activity by the identification of edematous areas associated with inflammation, early gadolinium technique can detect apical thrombus with high diagnostic accuracy and late gadolinium imaging allows the detection of endomyocardial fibrosis (Figure 1). The exclusive involvement of endomyocardium on LGE-CMR in conjunction with normal myocardial function, apical obliteration, and thrombus formation are typical findings in these patients ${ }^{36-38}$ highlighting the diagnostic role of CMR, a comprehensive tool for the early diagnosis of this rare but severe disease.

\section{Becker muscular dystrophy}

Becker muscular dystrophy is an X-linked recessive neuromuscular disorder that can be a rare cause of dilated cardiomyopathy. Cardiac myocytedystrophin deficiency leads to fiber necrosis causing biventricular replacement of myocardium with connective tissue or fat. ${ }^{39}$ The mid wall of the infero-lateral left ventricle is most commonly affected, with conduction system disease occurring late ${ }^{40}$ (Figure 1). In previous studies extensive mid-myocardial late gadolinium enhancement has been documented in patients with Becker dystrophy. ${ }^{41}$ More recently in two asymptomatic families with Becker muscular dystrophy, Mavrogeni et al. showed that left ventricular function was abnormal and the presence of subepicardial scar tissue was identified in the majority of them. ${ }^{42}$

\section{Conclusions}

CMR, using the available range of technique, is establishing itself as the gold standard non invasive test for determining the cardiac involvement in rare systemic diseases, adding prognostic value and guiding therapy. Progress is continuing and rapid with promising new techniques such as diffuse fibrosis assessment. As CMR becomes more widely available, more patients with rare diseases will have access to this important investigation.

\section{References}

1. Rehwald WG, Fieno DS, Chen EL, et al. Myocardial magnetic resonance imaging contrast agent concentrations after reversible and irreversible ischemic injury. Circulation 2002;105:224-9.

2. Flett AS, Hasleton J, Cook C, et al. Evaluation of techniques for the quantification of myocardial scar of differing etiology using cardiac magnetic resonance. J Am Coll Cardiol, 2011;4:150-6.

3. Fernandes JL, Pohost GM. Recent advances in cardiovascular magnetic resonance. Rev Cardiovasc Med 2011;12:e10712.

4. Hunninghake GW, Costabel U, Ando M, et al. ATS/ERS/WASOG statement on sarcoidosis. American Thoracic Society/ European Respiratory Society/World Association of Sarcoidosis and other Granulomatous Disorders. Sarcoidosis Vasc Diffuse Lung Dis 1999;16:149-73.

5. Sharma OP, Maheshwari A, Thaker K. Myocardial sarcoidosis. Chest 1993;103:
253-8.

6. Silverman KJ, Hutchins GM, Bulkley BH. Cardiac sarcoid a clinicopathologic study of 84 unselected patients with systemic sarcoidosis. Circulation 1978;58:1204-11.

7. Doughan AR, Williams BR. Cardiac sarcoidosis. Heart 2006;92:282-8.

8. Roberts WC, McAllister HA Jr., Ferrans VJ. Sarcoidosis of the heart. A clinicopathologic study of 35 necropsy patients (group 1) and review of 78 previously described necropsy patients (group 11). Am J Med 1977;63:86-108.

9. Serra JJ, Monte GU, Mello ES, et al. Images in cardiovascular medicine. Cardiac sarcoidosis evaluated by delayed-enhanced magnetic resonance imaging. Circulation 2003;107:e188-9.

10. Smedema JP, Snoep G, van Kroonenburgh MP, et al. Evaluation of the accuracy of gadolinium-enhanced cardiovascular magnetic resonance in the diagnosis of cardiac sarcoidosis. J Am Coll Cardiol 2005;45: 1683-90.

11. Smedema JP, Snoep G, van Kroonenburgh MP, et al. The additional value of gadolinium-enhanced MRI to standard assessment for cardiac involvement in patients with pulmonary sarcoidosis. Chest 2005;128: 1629-37.

12. Mahrholdt H, Wagner A, Judd RM, et al. Delayed enhancement cardiovascular magnetic resonance assessment of nonischaemic cardiomyopathies. Eur Heart J 2005;26:1461-74.

13. Yared K, Johri AM, Soni AV, et al. Cardiac sarcoidosis imitating arrhythmogenic right ventricular dysplasia. Circulation 2008;118:e113-5.

14. Schulz-Menger J, Wassmuth R, Abdel-Aty $\mathrm{H}$, et al. Patterns of myocardial inflammation and scarring in sarcoidosis as assessed by cardiovascular magnetic resonance. Heart 2006;92:399-400.

15. Vignaux 0, Dhote $\mathrm{R}$, Duboc $\mathrm{D}$, et al. Detection of myocardial involvement in patients with sarcoidosis applying T2weighted, contrast-enhanced, and cine magnetic resonance imaging: initial results of a prospective study. J Comput Assist Tomogr 2002;26:762-7.

16. Patel MR, Cawley PJ, Heitner JF, et al. Detection of myocardial damage in patients with sarcoidosis. Circulation 2009;120:1969-77.

17. Selvanayagam JB, Hawkins PN, Paul B, et al. Evaluation and management of the cardiac amyloidosis. J Am Coll Cardiol 2007;50:2101-10.

18. Maceira AM, Joshi J, Prasad SK, et al. Cardiovascular magnetic resonance in cardiac amyloidosis. Circulation 2005;111: 186-93.

19. Cheng AS, Banning AP, Mitchell AR, et al. 
Cardiac changes in systemic amyloidosis: visualisation by magnetic resonance imaging. Int J Cardiol 2006;113:E21-3.

20. Karamitsos TD, Piechnik SK, Banypersad SM, et al. Noncontrast tl mapping for the diagnosis of cardiac amyloidosis. JACC Cardiovasc Imaging 2013;6:488-97.

21. Banypersad SM, Sado DM, Flett AS, et al. Quantification of myocardial extracellular volume fraction in systemic AL amyloidosis: an equilibrium contrast cardiovascular magnetic resonance study. Circ Cardiovasc Imaging 2013;6:34-9.

22. Syed IS, Glockner JF, Feng D, et al. Role of cardiac magnetic resonance imaging in the detection of cardiac amyloidosis. JACC Cardiovasc Imaging 2010;3:155-64.

23. Maceira A, Prasad S, Hawkins P, et al. Cardiovascular magnetic resonance and prognosis in cardiac amyloidosis. J Cardiovasc Magn Reson 2008;10:54.

24. Wassmuth R, Abdel-Aty H, Bohl S, SchulzMenger J. Prognostic impact of T2-weighted CMR imaging for cardiac amyloidosis. Eur Radiol 2011;21:1643-50.

25. Steen VD and Medsger AT. Severe organ involvement in systemic sclerosis with diffuse scleroderma. Arthritis Rheum 2000;43:2437-44.

26. Tzelepis GE, Kelekis NL, Plastiras SC, et al. Pattern and distribution of myocardial fibrosis in systemic sclerosis: a delayed enhanced magnetic resonance imaging study. Ann Rheum Dis 2009;68:1878-84.

27. Hachulla AL, Launay D, Gaxotte V, et al. Cardiac magnetic resonance imaging in systemic sclerosis: a cross-sectional observational study of 52 patients. Ann Rheum Dis 2009;68:1878-84.

28. Clarke JT. Narrative review: Fabry disease. Ann Intern Med 2007;146:425-33.

29. Sado DM, White SK, Piechnik SK, et al. The identification and assessment of Anderson Fabry disease by cardiovascular magnetic resonance non-contrast myocardial T1 mapping. Circ Cardiovasc Imaging 2013. [Epub Ahead of Print].

30. Moon JC, Sheppard M, Reed E, et al. The histological basis of late gadolinium enhancement cardiovascular magnetic resonance in a patient with AndersonFabry disease. J Cardiovasc Magn Reson 2006;8:479-82.

31. Sachdev B, Takenaka T, Teraguchi H, et al. Prevalence of Anderson-Fabry disease in male patients with late onset hypertrophic cardiomyopathy. Circulation 2002;105: 1407-11.

32. Frustaci A, Chimenti C, Ricci R, et al. Improvement in cardiac function in the cardiac variant of Fabry's disease with galactose infusion therapy. N Engl J Med 2001;345:25-32.

33. Hughes DA, Elliott PM, Shah J, et al. Effects of enzyme replacement therapy on the cardiomyopathy of Anderson-Fabry disease: a randomised, double-blind, placebocontrolled clinical trial of agalsidasealfa. Heart 2008;94:153-8.

34. Weidemann F, Niemann M, Breunig F, et al. Long-term effects of enzyme replacement therapy on Fabry cardiomyopathy: evidence for a better outcome with early treatment. Circulation 2009;119:524-9.

35. Moon JC, Mundy HR, Lee PJ, et al. Images in cardiovascular medicine. Myocardial fibrosis in glycogen storage disease type III. Circulation 2003;107:e47.

36. Genee 0, Fichet J, Alison D. Images in cardiovascular medicine: cardiac magnetic resonance imaging and eosinophilic endomyocardial fibrosis. Circulation 2008; 118:e710-1.

37. Alvarez-Sala R, Prados C, Armada E, et al. Congestive cardiomyopathy and endobronchial granulomas as manifestations of Churg-Strauss syndrome. Postgrad Med J 1995;71:365-6.

38. Wassmuth R, Göbel U, Natusch A et al. Cardiovascular magnetic resonance imaging detects cardiac involvement in ChurgStrauss syndrome. J Cardiac Fail 2008;14: 856-60.

39. Muntoni F. Cardiomyopathy in muscular dystrophies. Curr Opin Neurol 2003;16: 577-83.

40. Finsterer J, Stollberger C. The heart in human dystrophinopathies. Cardiology 2003;99:1-19.

41. Varghese A, Pennell DJ. Late gadolinium enhanced cardiovascular magnetic resonance in Becker muscular dystrophy. Heart 2004;90:e59.

42. Mavrogeni S, Papavasiliou A, Skouteli E, et al. Cardiovascular magnetic resonance imaging evaluation of two families with Becker muscular dystrophy. Neuromuscul Disord 2010;20:717-9. 